\title{
Diversity of supernovae la determined using equivalent widths of Si II 4000
}

\author{
V. Arsenijevic, S. Fabbro, A. M. Mourão, and A. J. Rica da Silva \\ CENTRA - Centro Multidisciplinar de Astrofísica, IST, Avenida Rovisco Pais, 1049 Lisbon, Portugal \\ e-mail: arsenije@ist.utl.pt
}

Received 25 July 2008/ Accepted 17 September 2008

\begin{abstract}
Aims. Spectroscopic and photometric properties of low and high-z supernovae Ia (SNe Ia) have been analyzed in order to achieve a better understanding of their diversity and to identify possible SN Ia sub-types.

Methods. We use wavelet transformed spectra in which one can easily measure spectral features. We investigate the Si II 4000 equivalent width $\left(E W_{w}\{\mathrm{Si} I \mathrm{I}\}\right)$. The ability and, especially, the ease in extending the method to $\mathrm{SNe}$ at high- $z$ is demonstrated.

Results. We applied the method to $110 \mathrm{SNe}$ Ia and found correlations between $E W_{w}\{\mathrm{Si} \mathrm{II}\}$ and parameters related to the light-curve shape for 88 supernovae with available photometry. No evidence for evolution of $E W_{w}\{\mathrm{Si} \mathrm{II}\}$ with redshift is seen. Three sub-classes of SNe Ia were confirmed using an independent cluster analysis with only light-curve shape, colour, and $E W_{w}\{\mathrm{Si}$ II $\}$.

Conclusions. SNe from high- $z$ samples seem to follow a similar grouping to nearby objects. The $E W_{w}\{\mathrm{Si}$ II $\}$ value measured on a single spectrum may point towards SN Ia sub-classification, avoiding the need for expansion velocity gradient calculations.
\end{abstract}

Key words. supernovae: general - methods: data analysis

\section{Introduction}

The peak luminosities of type Ia supernovae (SNe Ia) are one of the best distance indicators at high redshifts. Consequently, due to the relatively small dispersion in their light-curves, they are used for cosmological parameter estimation (e.g., Riess et al. 1998; Permutter et al. 1999). However, some SNe seem to depart from standard behaviour and deserve further attention. The most representative examples are the sub-luminous $1991 \mathrm{bg}$-like objects, the unusual SNe 2002ic and $2005 \mathrm{gj}^{1}$, and the even more outstanding SN 2003fg (Howell et al. 2006). The existence of a single family of SNe Ia is thus still debatable (see Filippenko 1997, for a review on SN (in)homogeneity). Hence separating sub-types of $\mathrm{SNe}$ Ia or attempting a continuous parametrization of spectra and/or light-curves including the full diversity of SNe Ia observed up to now could reduce the scatter in the Hubble diagram and improve their use in cosmology.

In order to quantify the spectral differences between $\mathrm{SNe}$ Ia, Nugent et al. (1995) proposed a ratio between the depths of absorption features of Si II at $5972 \AA$ and $6355 \AA$. This ratio was also found to correlate with the absolute magnitude of SNe Ia and the light-curve shape parameter. We further investigate this idea exploring a consistent method of measuring SN features on transformed spectra using wavelets as will be explained below. One of the major concerns regarding the use of SNe Ia in cosmology is a possible systematic difference between the low- $z$ and high- $z$ samples (Blondin et al. 2006; Garavini et al. 2007a; Bronder et al. 2008). Reasons for such evolution are generally thought to be related to changes in metallicity or composition

\footnotetext{
${ }^{1}$ Some authors argue that SN 2002ic may be a type Ic supernova instead of Ia (e.g. Benetti et al. 2006; Wang et al. 2004), and the SN 2005gj classification as type Ia/IIn is still doubtful (Prieto et al. 2007; Trundle et al. 2008).
}

of the progenitor or circumstellar medium, progenitor mass or delay times. It is already known that progenitor age is a relevant issue for variability in SN peak luminosity (Prieto et al. 2008; Gallagher et al. 2005) and that metallicity might evolve with redshift (see recent work of Ellis et al. 2008, and reference therein). We shall demonstrate that the equivalent width of the Si II 4000 feature, $E W_{w}\{\mathrm{Si}$ II $\}$ defined below, coupled with lightcurve parameters, is an indicator of possible sub-classes within $\mathrm{SNe}$ Ia, consistent for both low and high- $z \mathrm{SNe}$.

\section{The Si II 4000 spectral feature}

The idea behind the use of spectral features to study supernovae relies on the possibility of defining normalized spectral ratios of these features that are common for all SNe. It has been demonstrated (Nugent et al. 1995; Folatelli 2004; Benetti et al. 2005; Bronder et al. 2008) that the ratios of the line depths or strengths are correlated with the light-curve shape. Here we analyze the possibility of extracting from spectral features additional information on intrinsic SN properties and their potential to distinguish SN sub-classes at both low and high-z.

For instance, the Si II $\lambda 6355$ absorption feature near $6150 \AA$ is widely used, being the most characteristic feature of a SN Ia. Available high- $z$ spectra however rarely extend to a rest-frame wavelength of $6150 \AA$, thus we are forced to focus our attention on the bluer part of the spectra, preferably the features Ca II H\&K or Si II 4000 in order to compare the nearby with the high- $z$ sample (Folatelli 2004).

The rest-frame peak flux at the time of maximum light occurs at about $4000 \AA$. At earlier epochs it is shifted slightly to lower wavelengths, while at later epochs the spectrum peaks at longer wavelengths. We are particularly interested in this spectral range 
since almost all high- $z$ spectra exhibit the Si II $\lambda 4130$ feature blueshifted to $4000 \AA$, which seems to be a good choice for comparison with low- $z$ SNe. It might serve well in distinguishing SNe Ia from SNe Ib and Ic when the usual Si II 6150 feature is not available, as it is often the case with high- $z \mathrm{SNe}$. The Si II 4000 feature is mostly located between $3900 \AA$ and $4200 \AA$ in the rest-frame. Reddening effects should also be diminished since this feature is narrow. Considering all these interesting properties, the feature is a good candidate to be used for cosmology (Bronder et al. 2008).

The intensity of the Si II 4000 feature is however often low and contamination can be important. To study this feature we apply a simple wavelet denoising procedure as described below.

\subsection{Discrete wavelet transform}

Wavelets are considered as a suitable tool for studying local properties of a signal due to their specific structure. Indeed, their local frequency representation allows us to process the data at different resolutions or scales. Therefore any local defect of a signal does not affect the whole wavelet decomposition. A local inhomogeneity in the SN spectrum, such as remaining residuals after subtraction of the host galaxy spectrum, sky emission, atmospheric absorption that is not completely removed in the reduction process, or any calibration or instrumental undesirable effect, affects just the coefficients in the wavelet decomposition in the small region where it appears.

To provide a homogeneous sample of data, we use spectra in the interval 3400-7000 $\AA$. Other choices of intervals do not make much difference due to the local character of the wavelet transform. All spectra were deredshifted first. Mallat's pyramid algorithm (Mallat 1989) is then performed to obtain the Discrete Time Wavelet Transform (DTWT) using Daubechies' extremal phase wavelets with 4 vanishing moments (D8) as a basis.

The number of decomposition levels refers to the number of levels of smoothed data and wavelet coefficients. The coefficients at the lower levels carry "high-frequency" information. If a spectrum consists of $N=2^{m}$ discrete points (which can be assumed without loss of generality), there will be $m$ wavelet coefficient bands and a scaling value, whose inverses are given in the decomposition shown in Fig. 1 (see Nason \& Silverman 1994).

It is often reasonable to assume that only a few large coefficients contain relevant information about the underlying signal, while small wavelet coefficients, especially from low scales, can be attributed to noise or any other undesirable high frequency feature. Thus this part of spectra, the scales 1-6 (see Fig. 1), will be excluded from our considerations. This step assures that after taking the inverse DTWT of such a spectrum, the resulting wavelet transformed spectrum becomes much smoother, and one can easily measure the features.

The analysis of the wavelet power spectrum ${ }^{2}$ revealed that scale 15 shows much greater discrepancy among $\mathrm{SNe}$, even for spectroscopically similar events. For this reason, scale 15 will not be included in the wavelet reconstruction of the spectra. We can support this step by the following technical explanation: the contribution from scale 15, shown in Fig. 1, is obtained by the inverse wavelet transform of a scaling constant, equal to the sample mean multiplied by the square root of the number of original data points, and the featureless inverse of the wavelet coefficient from the largest scale. The subtraction of the largest scale, as also

\footnotetext{
${ }^{2}$ Obtained by summing the squares of the wavelet coefficients, divided by the number of corresponding coefficients for each scale.
}
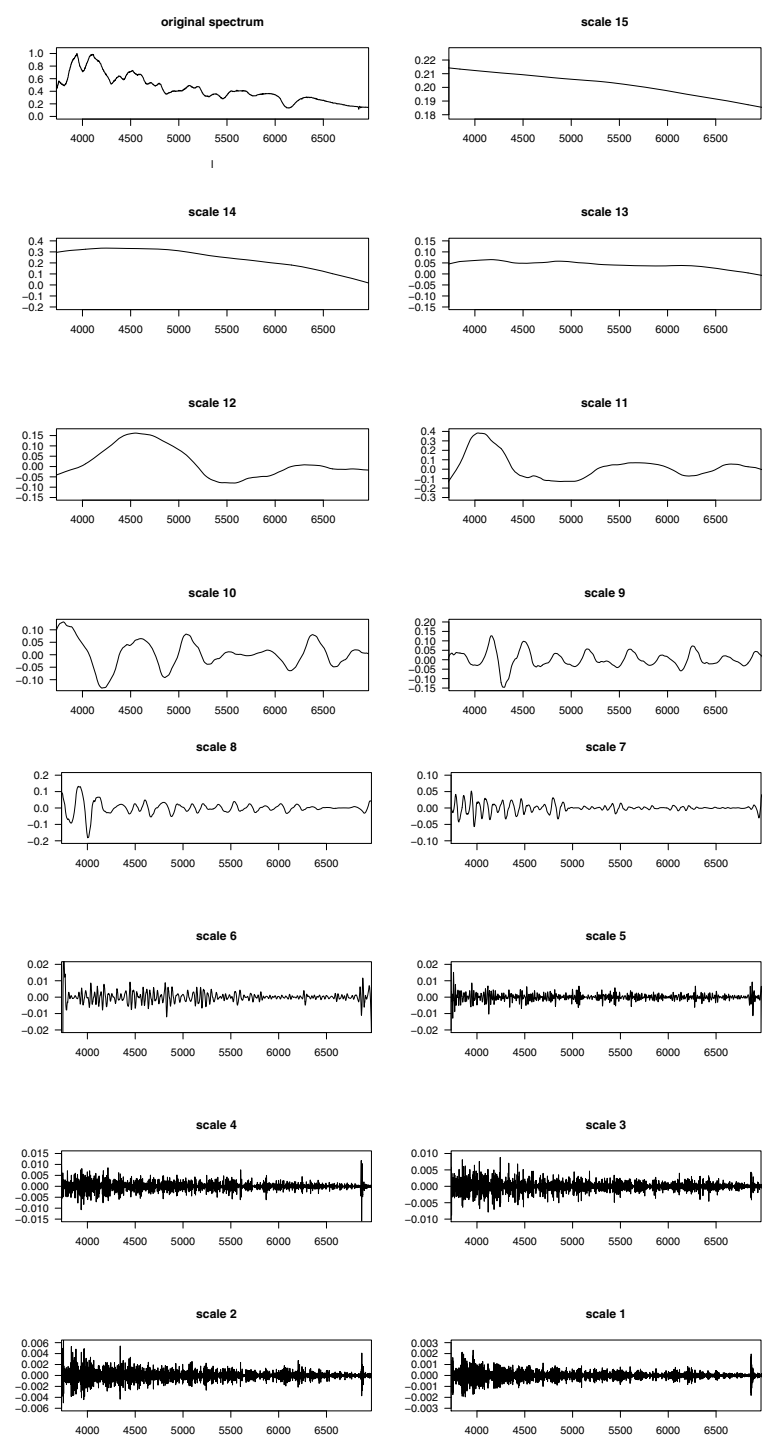

Fig. 1. Inverse wavelet reconstruction of separate scales of the wavelet decomposition of a spectrum of SN 1994D. The sum of contributions from scales 7-14 is used to construct the wavelet transformed spectra, $F_{w}(\lambda)$, used in this work.

mentioned in Starck et al. (1997), does not significantly deform the features of the spectrum.

After removal of scales 1-6 and scale 15 in the wavelet space, we apply the inverse DTWT. We treat all SNe Ia the same way to obtain a homogeneous sample of normalized wavelet transformed spectra. One could also apply a standard (soft) thresholding, but an optimal choice for a threshold value that cuts off all smaller wavelet coefficients and is consistent for whole variety of SN spectra is a complex issue and we have not attempted its implementation. Most spectral features analyses include a boxcar or Gaussian smoothing. Residual sky lines or galactic emission lines are thus also smoothed leading to possible misestimations of feature bounds and extrema. Our approach greatly reduces this effect.

\subsection{Determination of $E W_{w}\{$ Si Il $\}$}

In order to quantify SN Ia diversity with spectral features, rather than looking at deviation from the average SN Ia spectrum (James et al. 2006), some authors use a pseudo-equivalent-width 


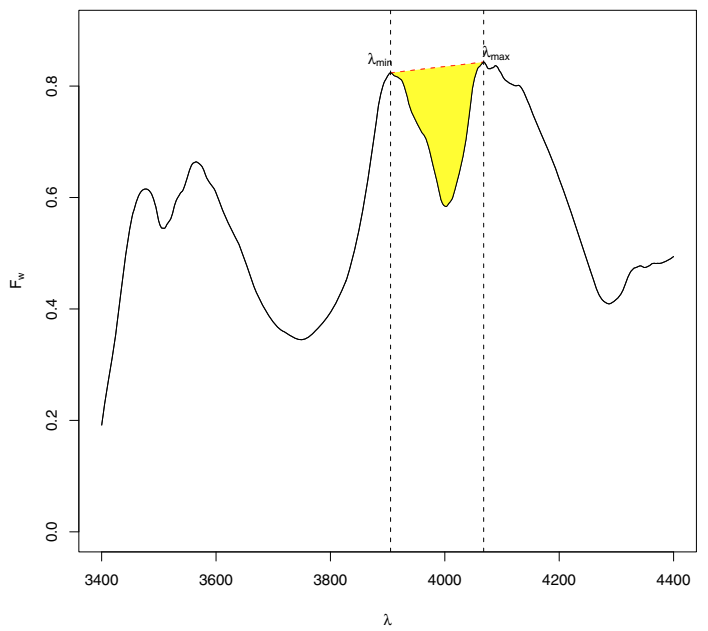

Fig. 2. Illustration of the $E W_{w}\{\mathrm{Si}$ II $\}$ definition on a wavelet transformed spectrum, $F_{w}(\lambda)$, of SN 1994D.

$(E W)$ (Hachinger et al. 2006; Garavini et al. 2007a; Bronder et al. 2008). Here, we apply the same definition but on the wavelet transformed spectra:

$E W_{w}=\sum_{i=1}^{N} \frac{F_{w}^{c}\left(\lambda_{i}\right)-F_{w}\left(\lambda_{i}\right)}{F_{w}^{c}\left(\lambda_{i}\right)} \Delta \lambda$,

where $F_{w}(\lambda)$ is the transformed SN flux after the wavelet procedure that has been performed on SN spectra, as described in 2.1 (therefore the subscript $w$ ); $F_{w}^{c}(\lambda)$ stands for the pseudocontinuum of the transformed flux, $N$ is the number of data points; the bounds of the feature are $\lambda_{\min }=\lambda_{1}$ and $\lambda_{\max }=\lambda_{N}$.

$E W_{w}\{\mathrm{Si}$ II $\}$ is defined as the $E W$ of Si II $\lambda 4130$ feature on transformed spectra,

$E W_{w}\{$ Si II $\} \equiv E W_{w}\{$ Si II $\lambda 4130\}$.

To determine the pseudo-continuum bounds, we use a multiple Gaussian peak-fitting semi-automated routine. All the pseudo$E W$ definitions used in the literature, including ours, depend on the accuracy of the pseudo-continuum bounds, and therefore on the spectrum signal-to-noise ratio $(S / N)$. The traditional definition of $E W$ does alleviate the dependency on $S / N$, but requires the full spectrum continuum to be well defined, which is more difficult for the SNe Ia. We are currently investigating this subject. We elaborate on the $S / N$ dependency in Sect. 2.3. We also note that our wavelet-based method for the removal of high-frequency features adds extra robustness to the pseudo- $E W$ estimation.

The $E W_{w}\{\mathrm{Si}$ II $\}$ evolves smoothly with the SN epoch, as we verified on a spectral series template given by the SALT2 model (Guy et al. 2007). We use the same prescription as Bongard et al. (2006) to obtain an estimate of $E W_{w}\{\mathrm{Si}$ III $\}$ at $t=0$ at maximum in $B$ : for a single epoch spectrum, we use the actual value (but increase the error), for two epoch spectra a straight line fit, and for multi-epoch spectra a quadratic polynomial fit.

We also estimate the statistical error made by the determination of extrema of the specific features considered, as both the wavelet transformed flux and the pseudo-continuum come with uncertainties that affect the calculated value of $E W_{w}$.

Standard error propagation, assuming small enough errors, applied on Eq. (1) leads to:

$\sigma_{\text {stat }}=\left[\sum_{i=1}^{N}\left(\frac{\sigma_{F_{w}}^{2}\left(\lambda_{i}\right)}{F_{w}^{c 2}\left(\lambda_{i}\right)}+\frac{F_{w}^{2}\left(\lambda_{i}\right)}{F_{w}^{c 4}\left(\lambda_{i}\right)} \sigma_{c}^{2}\left(\lambda_{i}\right)\right)(\Delta \lambda)^{2}\right]^{1 / 2}$,

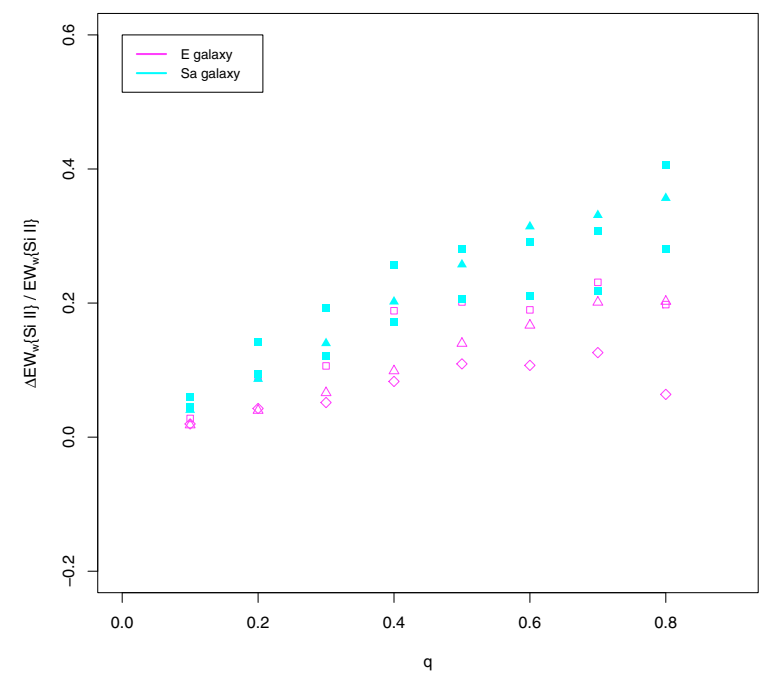

Fig. 3. Relative error of the measured $E W_{w}\{\mathrm{Si}$ II $\}$ as a function of $q$, the amount of host contamination for elliptical (open symbols) and spiral (filled symbols) galaxies for 3 low- $z$ SNe, 1981B, 1994D and 2002bo (given as triangle, diamond and square symbols respectively).

where $\sigma_{F_{w}}$ consists of the inverse $D T W T$ of the low scale coefficients from the wavelet decomposition that have been subtracted; $\sigma_{c}(\lambda)$ is obtained from the error of a straight line fit that defines the pseudo-continuum.

When we have multi-epoch spectra, $E W_{w}\{\mathrm{Si}$ II $\}$ is estimated for each of the epochs, and the error-weighted polynomial fit returns the propagated $\sigma_{\text {stat }}$. In the more difficult but common cases of single epoch spectra, we add to $\sigma_{\text {stat }}$ an error floor computed as the maximum dispersion observed on the epoch of the given spectrum. Typically we add $\sigma_{\text {floor }}=1.7$.

The dispersion seen in wavelengths of the feature bounds can be partly explained by redshift uncertainty, typically of 3-30 (respectively for $\Delta z$ of 0.001 and 0.01 ) at a redshift of 0.5 . However, no offset in $\lambda_{\min }$ or $\lambda_{\max }$ was found between good quality spectra and reduced $S / N$ data. The major reason for this is the use of the specific wavelet transform we perform on SN spectra to obtain the spectral function on which $E W_{w}$ s are measured. The main consequence is that statistical uncertainties in the high- $z$ set are generally higher due to lower $S / N$.

\subsection{Systematic errors}

To test the robustness of the $E W_{w}\{\mathrm{Si}$ II $\}$ measurements and quantify various systematic errors, we ran a set of simulations taking very high $S / N$ low- $z$ spectra on top of which we added extra contributions (for instance sky and/or galaxy) and Gaussian noise with a variable standard deviation.

One source of systematic errors is the host galaxy contamination. Very often, the light from the host galaxy is not perfectly removed and the remaining light affects the estimation of equivalent widths. We tested different amounts of galaxy contamination, up to $80 \%$ of the total integrated SN flux from 3400-7000 A. The simulations using template galaxy spectra of Hubble types $\mathrm{E}$ and $\mathrm{Sa}$ indicate that increasing galaxy contamination implies lower values of the measured $E W_{w}\{\mathrm{Si}$ II $\}$, as found in Garavini et al. (2007a); Bronder et al. (2008). In Fig. 3 we illustrate the correlation between increasing galaxy contamination level and the relative decrease in $E W_{w}\{\mathrm{Si}$ II $\}$.

Our results with a few $\mathrm{SNe}$ show a scatter that is generally of less than $20 \%$ for $\mathrm{SNe}$ in elliptical galaxies, even for 
Table 1. Summary of the error budget for the $E W_{w}\{\mathrm{Si}$ II $\}$ measurements.

\begin{tabular}{ll}
\hline \hline Source & Size \\
\hline statistical error & $15-27 \%$ (low- $z$ vs. high- $z$ ) \\
galaxy contamination & $<20-40 \%$ (E vs. S type) \\
low $S / N$ & $<5 \%$ for $S / N \geq 5$ \\
reddening & $<0.5 \%$ \\
high frequency feature residuals & $<0.5 \%$ \\
\hline
\end{tabular}

contaminations of 50-80\%, although it becomes greater for objects hosted in spiral galaxies, but never exceeds $40 \%$ of the true value. It may happen, however, that extremely high host-galaxy contamination distorts significantly the feature we measure, as in the case of the SN 2002bo spectrum with $80 \%$ elliptical galaxy contamination (see Fig. 3). Recall that Bronder et al. (2008) make a selection cut when galaxy contamination is found to be more than $65 \%$ since it can produce a change of up to $100 \%$ in the $E W$ value.

We expect that at low signal-to-noise ratios, spectra do not exhibit so clearly the Si II 4000 feature, making it difficult to measure accurately and possibly introducing a slight misestimation. Varying the noise in our simulations then measuring $E W_{w}\{\mathrm{Si} \mathrm{II}\}$ and corresponding error on each spectrum suggests that no significant bias is present in the $E W_{w}\{\mathrm{Si}$ II $\}$ values.

We also checked our hypothesis of the small effect of reddening on the Si II 4000 feature. Further simulations using the extinction law of Cardelli et al. (1989) with $R_{V}=3.1$ have shown that the relative error of $E W_{w}\{\mathrm{Si}$ II $\}$ measured on spectra with/without reddening correction never exceeds $0.5 \%$, while for $E W_{w}$ of Ca II H\&K reaches the order of $1 \%$. This fact supports the choice of the Si II 4000 feature instead of broader ones, such as $\mathrm{Ca}$ II $\mathrm{H} \& \mathrm{~K}$ or $\mathrm{Mg}$ II 4300 . Other extinction laws based on studies of dust properties in the Magellanic Clouds give similar results for the wavelength range under consideration (see, e.g. Pei 1992; Weingartner \& Draine 2001) and thus were not further investigated at this stage.

The contamination from residual sky line subtraction was also studied. To achieve this, we added to our set of several low- $z$ $\mathrm{SNe}$, which we redshifted at $z=0.5$, a fiducial sky spectrum and Gaussian noise. We verified that our DTWT procedure properly removes these lines, leaving $E W_{w}\{\mathrm{Si}$ II $\}$ unmodified.

In Table 1 we summarize all the measurement errors mentioned above.

\section{Data sets}

We then applied our measurement procedure to a set of published SN Ia data. The main selection criterion was the detection of the Si II spectral feature we are interested in. It thus depends on wavelength and epoch coverage. Tests performed on SN spectral templates indicate that it should be present within an interval of $[-15,15]$ days relative to $B$-maximum. All selected $\mathrm{SN}$ spectra have epochs within an interval of \pm 9 days around the maximum in $B$ in the rest-frame.

Furthermore, if the presence of strong host galaxy contamination was noticed resulting in severe misestimation of Si II 4000 feature, that spectrum was rejected. We mainly selected SNe with published photometry in order to check for correlations between $E W_{w}\{\mathrm{Si} \mathrm{II}\}$ and light-curve parameters.

\subsection{Low-z sample}

The analysis is applied to a sample of 35 local SNe taken from the literature and presented in Table 2. The spectroscopic data are taken mainly from public archives: SUSPECT ${ }^{3}$, CfA Supernova Archive ${ }^{4}$ and SUSPEND ${ }^{5}$. Spectra of $1991 \mathrm{~T}$ and 1991 bg-like SNe, also of other peculiar SN events were also considered. $E W_{w}\{\mathrm{Si} \mathrm{II}\}$ is measured on 124 spectra of low- $z \mathrm{SNe}$.

\subsection{High-z sample}

The principal difficulty when studying high- $z$ SNe, besides much noisier spectra compared to the low- $z$ events, is the wavelength coverage spanned by spectral observations resulting in the lack of major spectral features, like Si II 6150. However this is not the case with the Si II 4000 feature.

From the available set of high- $z$ SuperNova Legacy Survey (SNLS) data, we used $26 \mathrm{SNe}$ with spectral epochs within \pm 9 days relative to maximum in $B$ that have been published in Howell et al. (2005). This SN sample is distributed over $0.337<z<1.01$ (see Table 4). Usually there is only one spectrum for each high- $z$ supernova. We note that the host galaxy spectra were not subtracted.

High- $z$ spectra from the first two years of the ESSENCE project (see, e.g., Matheson et al. 2005; Miknaitis et al. 2007) were checked; among these, 26 spectra that show the presence of the Si II 4000 feature were used. No attempts to subtract the host galaxy from the spectra were made, but Matheson et al. (2005) did not report any strong contamination within this selected sample.

The Supernova Cosmology Project (SCP) high- $z$ spectra used in this work were provided by Hook et al. (2005) and Lidman et al. (2005), as seen from Table 4. Measurements were performed on 6 and 10 SN spectra respectively.

We use also 7 spectra from High- $z$ Supernova Search Team (HzSST) that were published in Tonry et al. (2003). Spectra from Hook et al. (2005) and Tonry et al. (2003) were corrected for host galaxy light.

Light-curve data come from Astier et al. (2006); Miknaitis et al. (2007); Permutter et al. (1999); Tonry et al. (2003); Kowalski et al. (2008).

\section{4. $E W_{w}\{\mathrm{Si} I I\}$ properties}

Armed with $E W_{w}\{\mathrm{Si}$ II $\}$ values for 32 low- $z$ and 75 high- $z$ $\mathrm{SNe}$ Ia, we searched for correlations with light-curve parameters. In order to apply a consistent procedure for all SNe, we fitted the available light-curves with the SALT2 model. We ended up with 30 low $-z$ and 58 high- $z$ SNe, for which $E W_{w}\{\mathrm{Si}$ II $\}$ and SALT2 parameters were calculated, as can be seen in Tables 3 and 4 . We also tried our analysis with published parameter values from the MLCS2k2 (Jha et al. 2007) and SALT (Guy et al. 2005; Kowalski et al. 2008) fitters.

\subsection{Relation with the light-curve parameters}

We first checked the correlation between $E W_{w}\{\mathrm{Si}$ II $\}$ and $x_{1}$ (SALT2 width parameter), illustrated in the top panel of Fig. 4.

\footnotetext{
3 http://bruford.nhn.ou.edu/ suspect/index 1.html

${ }^{4}$ http://cfa-www.harvard.edu/oir/Research/supernova/ SNarchive.html

5 http://www.nhn.ou.edu/ jeffery/astro/sne/spectra/ spectra.html
} 
Table 2. List of nearby SNe used in this work.

\begin{tabular}{|c|c|c|c|}
\hline SN name & $\begin{array}{c}\text { Epoch } \\
\text { (with respect to } B \text { maximum) }\end{array}$ & Bands & References \\
\hline 1981B & 0 & $U B V$ & 1 \\
\hline 1986G & $-3,-1,0,1,2$ & $B V$ & 2 \\
\hline 1989B & $-5,-1,0,3,5$ & $U B V R I$ & 3,4 \\
\hline $1990 \mathrm{~N}$ & 2,7 & $U B V R I$ & 5 \\
\hline $1991 \mathrm{M}$ & 3 & VRI & 6 \\
\hline 1992A & $-5,-1,3,5$ & $U B V R I$ & 7 \\
\hline 1994D & $-5,-4,-2,2,4$ & $U B V R I$ & 8 \\
\hline 1996X & $-2,0,1$ & $B V R I$ & 9 \\
\hline 1998aq & $0,1,2,3,4,5$ & $U B V R I$ & 10 \\
\hline 1998bu & $-3,-2,-1$ & $U B V R I$ & 11 \\
\hline 1999ee & $-4,-2,0,5$ & $U B V R I$ & 12 \\
\hline $2000 \mathrm{E}$ & $-3,-2$ & $U B V R I$ & 13 \\
\hline $2002 b f$ & 3 & $B V R I$ & 14 \\
\hline 2002bo & $-5,-4,-3,-2,-1,4$ & $U B V R I$ & 15 \\
\hline 2002er & $-5,-4,-3,-2,-1,0,2,4,5$ & $U B V R I$ & 16,17 \\
\hline $2003 \mathrm{cg}$ & $-5,-1,1,4$ & $U B V R I$ & 18 \\
\hline $2003 \mathrm{du}$ & $-4,-2,-1,0,1,3,4$ & UBVR & 19,20 \\
\hline $2004 d t$ & $-5,-4,-3,-2,-1,0,1,2,3,4$ & .. & 14,21 \\
\hline $2004 \mathrm{eo}$ & $-3,2$ & $U B V R I$ & 22 \\
\hline $2004 S$ & 1 & $U B V R I$ & 23 \\
\hline 2005am & $-1,2$ & $U B V$ & 24 \\
\hline $2005 \mathrm{cf}$ & $-4,-3,-1,0,4,5$ & $U B V R I$ & 25,26 \\
\hline 2005hj & $-5,0,2,3,5$ & $\cdots$ & 27 \\
\hline $2006 \mathrm{gz}$ & $-4,-1$ & $U B V r i$ & 28 \\
\hline \multicolumn{4}{|c|}{ SNe 91bg-like } \\
\hline 1991bg & 1,2 & $B V R I$ & 29 \\
\hline 1999by & $-5,-4,-3,3,4,5$ & UBVRI & 30 \\
\hline \multicolumn{4}{|c|}{ SNe 91T-like } \\
\hline 1991T & $-3,0$ & $U B V R I$ & $31,32,33$ \\
\hline $2000 \mathrm{cx}$ & $-3,-2,0,1,5$ & UBVRI & 34 \\
\hline \multicolumn{4}{|c|}{ SNe Ia peculiar } \\
\hline $1997 \mathrm{br}$ & -4 & $U B V R I$ & 35 \\
\hline $1997 \mathrm{cn}$ & 3 & $U B V R I$ & 36 \\
\hline 1999aа & $-3,-1$ & $U B V R I$ & 37 \\
\hline $1999 \mathrm{ac}$ & 0,2 & $U B V R I$ & 38,39 \\
\hline 1999aw & 3,5 & $B V R I$ & 40 \\
\hline $2002 \mathrm{cx}$ & $-4,-1$ & $B V R I$ & 41,42 \\
\hline $2005 \mathrm{hk}$ & $-5,-4,4$ & $U B V R I$ & 43,44 \\
\hline
\end{tabular}

References: (1) Branch et al. (1983); (2) Phillips et al. (1987); (3) Barbon et al. (1990); (4) Wells et al. (1994); (5) Mazzali et al. (1993); (6) Gomez \& Lopez (1998); (7) Kirshner et al. (1993); (8) Patat et al. (1996); (9) Salvo et al. (2001); (10) Branch et al. (2003); (11) Jha et al. (1999); (12) Hamuy et al. (2002); (13) Valentini et al. (2003); (14) Leonard et al. (2005); (15) Benetti et al. (2004); (16) Pignata et al. (2004); (17) Kotak et al. (2005); (18) Elias-Rosa et al. (2006); (19) Anupama et al. (2005); (20) Stanishev et al. (2007); (21) Altavilla et al. (2007); (22) Pastorello et al. (2007b); (23) Krisciunas et al. (2007); (24) Brown et al. (2005); (25) Garavini et al. (2007b); (26) Pastorello et al. (2007a); (27) Quimby et al. (2007); (28) Hicken et al. (2007); (29) Turatto et al. (1996); (30) Garnavich et al. (2004); (31) Jeffery et al. (1992); (32) Phillips et al. (1992); (33) Mazzali et al. (1995); (34) Li et al. (2001); (35) Li et al. (1999); (36) Turatto et al. (1998); (37) Garavini et al. (2004); (38) Garavini et al. (2005); (39) Phillips et al. (2006); (40) Strolger et al. (2002); (41) Li et al. (2003); (42) Branch et al. (2004); (43) Phillips et al. (2007); (44) Sahu et al. (2008).

The $x_{1}$ parameter measures the departure of the stretch of a SN from the average value of the training sample (in standard deviations); its average value is adopted to satisfy $\left\langle x_{1}\right\rangle=0$ and $\left\langle x_{1}^{2}\right\rangle=1$ (Guy et al. 2007). Nugent et al. (1995) and Hachinger et al. (2006) found a strong correlation between the ratio of the depth of the absorption features of Si II at 5972 and $6355 \AA$, $\mathcal{R}\left(\mathrm{Si}\right.$ II), and $E W\{\mathrm{Si}$ II $\lambda 5972\}$ and $\Delta m_{15}$. We confirm a similar correlation for low and high- $z$ SNe using the Si II 4000 feature and a different light-curve model (SALT2).

We noticed however several SNe departing from the main trend. A similar behaviour was highlighted by Benetti et al. (2005) using velocity gradients inferred from the measured blueshift of the Si II absorption feature at $6355 \AA$. The authors identified sub-classes of $\mathrm{SNe}$ in the low redshift sample termed: high velocity gradient (HVG), low velocity gradient (LVG) and FAINT SNe. The latter group includes those SNe that are found to have similar brightness as that of SN 1991bg. Other authors confirmed the same sub-classifications (see for instance Hachinger et al. 2006; Pastorello et al. 2007b). These authors also use the expansion velocity gradient of the Si II $\lambda 6355$ feature to classify $\mathrm{SNe}$, therefore multi-epoch spectra are required. A similar grouping was found by Branch et al. (2006) studying the equivalent widths of Si II $\lambda 6355$ and $\lambda 5972$ features. These approaches however are hardly applicable to high- $z$ SNe due to the wavelength coverage. 
Table 3. SALT2 estimates and $E W_{w}\{\mathrm{Si}$ II $\}$ results for nearby SNe. The blank spaces “...” in $E W_{w}\{\mathrm{Si}$ II $\}$ column indicate insufficient presence of $\mathrm{Si}$ II feature despite an adequate wavelength coverage.

\begin{tabular}{|c|c|c|c|c|c|}
\hline SN name & $z_{\mathrm{CMB}}$ & $m_{B}$ & $x_{1}$ & $\bar{c}$ & $\overline{E W_{w}\{\mathrm{Si} \mathrm{II}\}}$ \\
\hline 1981B & 0.0072 & $12.0074 \pm 0.0217$ & $-0.6660 \pm 0.1673$ & $0.1530 \pm 0.0196$ & $27.47 \pm 2.33$ \\
\hline $1986 \mathrm{G}$ & 0.0028 & $12.0184 \pm 0.0371$ & $-3.0859 \pm 0.3261$ & $0.9851 \pm 0.0409$ & $3.56 \pm 2.67$ \\
\hline 1989B & 0.0036 & $12.2424 \pm 0.0177$ & $-1.0569 \pm 0.1418$ & $0.4736 \pm 0.0141$ & $20.67 \pm 1.48$ \\
\hline $1990 \mathrm{~N}$ & 0.0045 & $12.6599 \pm 0.0138$ & $0.7208 \pm 0.1168$ & $0.0773 \pm 0.0121$ & $15.68 \pm 2.45$ \\
\hline $1991 M$ & 0.0076 & $14.3553 \pm 0.1376$ & $-1.5904 \pm 0.3325$ & $0.0036 \pm 0.1140$ & $28.53 \pm 2.31$ \\
\hline 1992A & 0.0059 & $12.5356 \pm 0.0100$ & $-2.0636 \pm 0.0995$ & $0.0810 \pm 0.0103$ & $30.37 \pm 2.42$ \\
\hline 1994D & 0.0027 & $11.7314 \pm 0.0095$ & $-2.1674 \pm 0.0842$ & $-0.0643 \pm 0.0100$ & $23.62 \pm 2.47$ \\
\hline 1996X & 0.0078 & $12.9832 \pm 0.0151$ & $-1.2519 \pm 0.1539$ & $0.0408 \pm 0.0153$ & $17.86 \pm 2.61$ \\
\hline 1998aq & 0.0042 & $12.2893 \pm 0.0070$ & $-0.4507 \pm 0.0615$ & $-0.0920 \pm 0.0076$ & $9.69 \pm 2.52$ \\
\hline 1998bu & 0.0042 & $12.0777 \pm 0.0062$ & $-0.3570 \pm 0.0556$ & $0.3320 \pm 0.0068$ & $12.95 \pm 1.37$ \\
\hline 1999ee & 0.0105 & $14.8268 \pm 0.0062$ & $0.4171 \pm 0.0526$ & $0.3052 \pm 0.0067$ & $10.91 \pm 2.09$ \\
\hline $2000 \mathrm{E}$ & 0.0048 & $12.8034 \pm 0.0099$ & $0.4851 \pm 0.1117$ & $0.2058 \pm 0.0101$ & $8.13 \pm 2.47$ \\
\hline $2002 \mathrm{bf}$ & 0.0247 & $16.2978 \pm 0.0445$ & $-0.4205 \pm 0.2040$ & $0.2293 \pm 0.0275$ & $29.29 \pm 2.32$ \\
\hline 2002bo & 0.0053 & $13.9533 \pm 0.0086$ & $-0.6595 \pm 0.0668$ & $0.4711 \pm 0.0090$ & $26.85 \pm 2.35$ \\
\hline $2002 \mathrm{er}$ & 0.0086 & $14.2263 \pm 0.0100$ & $-1.1062 \pm 0.0825$ & $0.1918 \pm 0.0102$ & $23.18 \pm 2.32$ \\
\hline $2003 \mathrm{cg}$ & .0041 & $15.7912 \pm 0.0089$ & $-0.4629 \pm 0.0655$ & $1.3005 \pm 0.0091$ & $20.69 \pm 2.40$ \\
\hline 2003du & 0.0064 & $13.4923 \pm 0.0093$ & $0.1035 \pm 0.0697$ & $-0.0472 \pm 0.0087$ & $11.33 \pm 0.94$ \\
\hline 2004dt & 0.0188 & .. & .. & $\cdots$ & $29.48 \pm 2.35$ \\
\hline $2004 \mathrm{eo}$ & 0.0147 & $4 \pm 0.0066$ & $-1.4892 \pm 0.0601$ & $1 \pm 0.0075$ & $20.03 \pm 4.19$ \\
\hline $2004 S$ & 0.0091 & .0293 & $-0.3168=$ & $0.1317 \pm 0.0166$ & 2.58 \\
\hline $2005 \mathrm{am}$ & 0.0090 & $13.7303 \pm 0.0225$ & $-2.6235 \pm 0.1490$ & $0.2102 \pm 0.0167$ & $13.40 \pm 3.09$ \\
\hline $2005 \mathrm{cf}$ & 0.0070 & $13.0841 \pm 0.0054$ & $-0.3598 \pm 0.0471$ & $-0.0387 \pm 0.0060$ & $14.70 \pm 1.53$ \\
\hline 2005hj & 0.0574 & 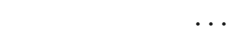 & $\ldots$ & $\cdots$ & $8.36 \pm 2.76$ \\
\hline $2006 \mathrm{gz}$ & 0.0277 & $15.7957 \pm 0.0176$ & $2.3344 \pm 0.1741$ & $0.0490 \pm 0.0148$ & $7.60 \pm 4.17$ \\
\hline \multicolumn{6}{|c|}{ SNe 91bg-like } \\
\hline 1991bg & 0.0042 & $14.6012 \pm 0.0381$ & $-2.7372 \pm 0.1940$ & $0.7185 \pm 0.0309$ & \\
\hline 1999by & 0.0029 & $13.6486 \pm 0.0143$ & $-3.0586 \pm 0.2231$ & $0.5483 \pm 0.0148$ & $10.80 \pm 4.38$ \\
\hline \multicolumn{6}{|c|}{ SNe 91T-like } \\
\hline $1991 \mathrm{~T}$ & 0.0070 & $11.5450 \pm 0.0118$ & $1.0398 \pm 0.1138$ & $0.2008 \pm 0.0121$ & $0.54 \pm 1.77$ \\
\hline $2000 \mathrm{cx}$ & 0.0079 & $13.0444 \pm 0.0082$ & $-1.3500 \pm 0.0790$ & $0.0604 \pm 0.0090$ & $13.12 \pm 2.92$ \\
\hline \multicolumn{6}{|c|}{ SNe Ia peculiar } \\
\hline $1997 \mathrm{br}$ & 0.0080 & $13.4941 \pm 0.0160$ & $0.0209 \pm 0.1319$ & $0.2849 \pm 0.0128$ & \\
\hline $1997 \mathrm{cn}$ & 0.0170 & $15.9641 \pm 0.1168$ & $-2.6791 \pm 0.3456$ & $0.1092 \pm 0.0753$ & $4.57 \pm 3.24$ \\
\hline 1999aа & 0.0144 & $14.7077 \pm 0.0131$ & $1.1371 \pm 0.1313$ & $-0.0242 \pm 0.0124$ & $3.19 \pm 2.66$ \\
\hline 1999ac & 0.0099 & $14.1078 \pm 0.0092$ & $-0.1542 \pm 0.1064$ & $0.1118 \pm 0.0102$ & $17.33 \pm 2.35$ \\
\hline 1999aw & 0.0393 & $16.7145 \pm 0.0163$ & $2.0927 \pm 0.1799$ & $-0.0069 \pm 0.0157$ & $1.08 \pm 1.54$ \\
\hline $2002 \mathrm{cx}$ & 0.0250 & $17.7281 \pm 0.0189$ & $0.1253 \pm 0.1507$ & $0.3253 \pm 0.0154$ & \\
\hline $2005 \mathrm{hk}$ & 0.0118 & $15.9015 \pm 0.0112$ & $-0.4887 \pm 0.1149$ & $0.3112 \pm 0.0111$ & $5.13 \pm 2.53$ \\
\hline
\end{tabular}

We distinguished exactly the same three sub-classes applying a hierarchical cluster analysis in a much smaller parameter space, using only $E W_{w}\{\mathrm{Si}$ II $\}$ with a SALT2 light-curve shape, $x_{1}$, and colour parameter.

We emphasize as an advantage of our method the ability to identify the 3 sub-classes with only one spectrum relatively close to $B$-maximum. If we remove the $E W_{w}\{\mathrm{Si}$ II $\}$ parameter from the cluster analysis we are not able to find the three clusters of $\mathrm{SNe}$. We also ran the cluster analysis on low- $z \mathrm{SNe}$ using other estimates of light-curve width such as $\Delta m_{15}$, stretch, $\Delta$ (MLCS) and identified the same three sub-classes.

It is known that for $\mathrm{HVG}^{6} \mathrm{SNe}$ the Si II $\lambda 6355$ line evolves rapidly (Benetti et al. 2005). These SNe generally have larger photospheric velocities than SNe with a slower evolution of the Si II $\lambda 6355$ feature, such as the LVG SNe. This latter group includes both normal SNe Ia and the brightest ones. Again, in the classification of Branch et al. (2006), the LVG group corresponds to core-normals and shallows. However, SNe from both HVG and LVG groups have similar maximum luminosities. The

\footnotetext{
6 This group corresponds to the broad-line group in the classification of Branch et al. (2006).
}

difference in photospheric velocity comes as a consequence of the difference in photospheric temperature; the HVG have a lower temperature compared to LVG SNe.

There are also a few SNe, namely 1983G, 1984A, 2002bf and 2004dt, that show similar behaviour to that of SN 2002bo, a well studied HVG supernova. The similarities between these SNe were also pointed out by Altavilla et al. (2007) and Leonard et al. (2005). All of them belong to the group of SNe with unusually high photospheric velocities. In addition, 2004dt and 2002 bf are both highly polarized. Spectropolarimetry can provide, in general, a probe of supernova geometry; greater divergence from spherical symmetry normally causes a higher polarization, but the latter may also be caused by clumping (see Wang $\&$ Wheeler 2008, for the latest review). Furthermore, Wang et al. (2007) found significant peculiarity of SN 2004dt comparing the degree of polarization across the Si II $\lambda 6355$ line and light-curve decline parameter $\Delta m_{15}$.

The difference between HVG and LVG SNe can also be studied in objects that have similar decline rates, as shown in Tanaka et al. (2008) for SNe 2002bo and 2001el. They conclude that burning in LVG is less powerful than in HVG SNe, thus there is a 
Table 4. SALT2 estimates and $E W_{w}\{\mathrm{Si}$ II $\}$ for high- $z$ sample.

\begin{tabular}{|c|c|c|c|c|c|c|}
\hline SN name & $z_{\mathrm{CMB}}$ & $\bar{m}$ & $x_{1}$ & $c$ & $E W_{w}\{\mathrm{Si} \mathrm{II}\}$ & Reference \\
\hline 03D1ax & 0.496 & $22.9691 \pm 0.0160$ & $-1.0045 \pm 0.1715$ & $-0.0304 \pm 0.0285$ & $9.01 \pm 3.95$ & 1 \\
\hline 03D1bk & 0.865 & $\ldots$ & $\ldots$ & & $13.50 \pm 5.36$ & 1 \\
\hline 03D1co & 0.68 & $24.1069 \pm 0.0481$ & $0.9602 \pm 0.6335$ & $-0.0229 \pm 0.0539$ & $8.21 \pm 5.94$ & 1 \\
\hline 03D1ew & 0.868 & $24.3489 \pm 0.0576$ & $0.4624 \pm 0.4097$ & $-0.0762 \pm 0.1830$ & $9.94 \pm 8.66$ & 1 \\
\hline 03D1fq & 0.80 & $24.5299 \pm 0.0343$ & $-1.8585 \pm 0.5215$ & $-0.2695 \pm 0.1905$ & $31.30 \pm 4.38$ & 1 \\
\hline 03D4cn & 0.818 & $24.6580 \pm 0.0401$ & $-2.3380 \pm 0.6214$ & $0.1416 \pm 0.1758$ & $17.25 \pm 4.15$ & 1 \\
\hline 03D4cy & 0.9271 & $24.6993 \pm 0.0873$ & $0.2584 \pm 0.4974$ & $-0.3657 \pm 0.1862$ & $21.11 \pm 8.40$ & 1 \\
\hline 03D4cz & 0.695 & $24.0406 \pm 0.0413$ & $-2.3515 \pm 0.4144$ & $-0.0699 \pm 0.0605$ & $6.09 \pm 4.62$ & 1 \\
\hline 03D4fd & 0.791 & $24.2177 \pm 0.0248$ & $-0.1670 \pm 0.4221$ & $-0.0418 \pm 0.0527$ & $23.54 \pm 6.08$ & 1 \\
\hline 03D4gl & 0.56 & $23.1869 \pm 0.0428$ & $-0.8379 \pm 0.5806$ & $-0.0111 \pm 0.0426$ & $5.58 \pm 6.56$ & 1 \\
\hline 04D1de & 0.7677 & $\cdots$ & $\cdots$ & $\cdots$ & $5.73 \pm 5.24$ & 1 \\
\hline 04D1hd & 0.3685 & $\cdots$ & $\cdots$ & $\cdots$ & $15.86 \pm 3.87$ & 1 \\
\hline 04D3dd & 1.01 & $25.0592 \pm 0.1625$ & $1.1167 \pm 0.6553$ & $-0.1155 \pm 0.2196$ & $44.42 \pm 8.38$ & 1 \\
\hline 04D3fq & 0.73 & $24.1009 \pm 0.0262$ & $-0.7710 \pm 0.4011$ & $-0.0168 \pm 0.0480$ & $14.31 \pm 7.25$ & 1 \\
\hline 04D3gx & 0.91 & $24.6957 \pm 0.0732$ & $-0.1806 \pm 0.5141$ & $-0.1816 \pm 0.1746$ & $29.07 \pm 10.80$ & 1 \\
\hline 04D3kr & 0.3373 & $21.9321 \pm 0.0207$ & $1.0967 \pm 0.1851$ & $0.0131 \pm 0.0167$ & $10.69 \pm 4.27$ & 1 \\
\hline 04D3lp & 0.983 & $24.8964 \pm 0.1414$ & $-1.4766 \pm 0.5405$ & $0.1080 \pm 0.2277$ & $20.86 \pm 6.11$ & 1 \\
\hline 04D3mk & 0.813 & $\cdots$ & $\cdots$ & $\cdots$ & $4.70 \pm 4.37$ & 1 \\
\hline 04D3ml & 0.95 & $24.5060 \pm 0.0725$ & $1.4300 \pm 0.7394$ & $0.0824 \pm 0.1447$ & $10.99 \pm 8.40$ & 1 \\
\hline 04D3nh & 0.3402 & $22.0925 \pm 0.0161$ & $0.5018 \pm 0.1758$ & $0.0350 \pm 0.0148$ & $20.07 \pm 4.24$ & 1 \\
\hline 04D3ny & 0.81 & $24.3118 \pm 0.0418$ & $2.4869 \pm 1.2655$ & $-0.1175 \pm 0.1758$ & $8.91 \pm 6.51$ & 1 \\
\hline 04D4dm & 0.811 & $24.3974 \pm 0.0404$ & $0.8626 \pm 1.1636$ & $-0.1282 \pm 0.1661$ & $11.50 \pm 7.09$ & 1 \\
\hline 04D4hu & 0.7027 & $\cdots$ & $\cdots$ & $\cdots$ & $14.21 \pm 3.89$ & 1 \\
\hline 04D4ic & 0.68 & $\cdots$ & $\cdots$ & $\cdots$ & $15.33 \pm 4.63$ & 1 \\
\hline 04D4ii & 0.866 & $\cdots$ & $\cdots$ & $\cdots$ & $14.24 \pm 4.74$ & 1 \\
\hline 04D4jy & 0.93 & $\cdots$ & $\cdots$ & $\cdots$ & $22.66 \pm 11.59$ & 1 \\
\hline b010 & 0.591 & $23.4054 \pm 0.0790$ & $1.9986 \pm 0.9094$ & $-0.1171 \pm 0.0948$ & $6.16 \pm 3.17$ & 2 \\
\hline b013 & 0.426 & $22.6230 \pm 0.0427$ & $-0.5961 \pm 0.4047$ & $0.0244 \pm 0.0524$ & $32.85 \pm 2.88$ & 2 \\
\hline b016 & 0.329 & $22.5340 \pm 0.1385$ & $0.2256 \pm 0.7683$ & $0.2086 \pm 0.1276$ & $7.50 \pm 3.21$ & 2 \\
\hline b020 & 0.425 & $22.4605 \pm 0.2181$ & $-1.4169 \pm 1.8301$ & $0.0434 \pm 0.1987$ & $13.51 \pm 2.72$ & 2 \\
\hline $\mathrm{d} 033$ & 0.531 & $23.0873 \pm 0.0802$ & $3.8152 \pm 1.1886$ & $-0.2914 \pm 0.0986$ & $18.13 \pm 3.92$ & 2 \\
\hline $\mathrm{d} 058$ & 0.583 & $23.5307 \pm 0.0418$ & $0.1674 \pm 0.6575$ & $0.1711 \pm 0.0754$ & $36.00 \pm 3.91$ & 2 \\
\hline $\mathrm{d} 083$ & 0.333 & $21.0031 \pm 0.0333$ & $1.2251 \pm 0.2224$ & $-0.0512 \pm 0.0349$ & $9.39 \pm 2.64$ & 2 \\
\hline d085 & 0.401 & $22.4501 \pm 0.0480$ & $0.6541 \pm 0.3903$ & $0.0428 \pm 0.0590$ & $13.87 \pm 2.99$ & 2 \\
\hline d093 & 0.363 & $21.8967 \pm 0.0480$ & $1.0120 \pm 0.2783$ & $-0.0994 \pm 0.0482$ & $7.89 \pm 7.35$ & 2 \\
\hline d117 & 0.309 & $22.3667 \pm 0.0997$ & $-1.8445 \pm 0.3583$ & $0.0968 \pm 0.0916$ & $21.98 \pm 2.76$ & 2 \\
\hline d149 & 0.342 & $22.0957 \pm 0.0455$ & $0.3705 \pm 0.3584$ & $0.0109 \pm 0.0436$ & $9.52 \pm 3.10$ & 2 \\
\hline e020 & 0.159 & $21.0616 \pm 0.1498$ & $0.0291 \pm 0.3768$ & $0.3722 \pm 0.1266$ & $13.18 \pm 2.35$ & 2 \\
\hline e029 & 0.332 & $22.4054 \pm 0.0803$ & $-2.3762 \pm 1.0506$ & $0.0532 \pm 0.0810$ & $15.36 \pm 2.60$ & 2 \\
\hline e108 & 0.469 & $22.5728 \pm 0.0815$ & $2.0057 \pm 1.5990$ & $-0.1079 \pm 0.0887$ & $14.85 \pm 3.32$ & 2 \\
\hline e132 & 0.239 & $21.7710 \pm 0.0645$ & $-0.3371 \pm 0.2480$ & $0.3003 \pm 0.0605$ & $21.33 \pm 2.47$ & 2 \\
\hline e138 & 0.612 & $23.7475 \pm 0.0501$ & $-3.8738 \pm 0.7231$ & $0.2588 \pm 0.0996$ & $18.40 \pm 3.21$ & 2 \\
\hline e147 & 0.645 & $23.3283 \pm 0.0463$ & $-0.3841 \pm 0.4340$ & $-0.1072 \pm 0.0570$ & $18.86 \pm 2.90$ & 2 \\
\hline e148 & 0.429 & $22.6329 \pm 0.0335$ & $-1.1050 \pm 0.3499$ & $-0.0489 \pm 0.0448$ & $15.42 \pm 2.66$ & 2 \\
\hline f011 & 0.539 & $23.2794 \pm 0.0363$ & $-1.4498 \pm 0.4991$ & $0.0598 \pm 0.0620$ & $23.81 \pm 2.91$ & 2 \\
\hline f041 & 0.561 & $23.1138 \pm 0.0479$ & $2.4526 \pm 1.2001$ & $-0.0763 \pm 0.0650$ & $15.07 \pm 3.00$ & 2 \\
\hline f076 & 0.410 & $22.3460 \pm 0.0641$ & $-1.0001 \pm 0.6384$ & $0.0600 \pm 0.0899$ & $15.94 \pm 3.44$ & 2 \\
\hline f096 & 0.412 & $22.8398 \pm 0.2053$ & $0.1328 \pm 2.0466$ & $0.2771 \pm 0.1320$ & $8.35 \pm 2.76$ & 2 \\
\hline $\mathrm{f} 216$ & 0.599 & $23.7736 \pm 0.0775$ & $-3.2479 \pm 1.0666$ & $-0.0383 \pm 0.1316$ & $12.44 \pm 3.15$ & 2 \\
\hline $\mathrm{f} 231$ & 0.619 & $23.4465 \pm 0.0355$ & $1.2394 \pm 0.3607$ & $-0.0759 \pm 0.0623$ & $17.03 \pm 3.30$ & 2 \\
\hline $\mathrm{f} 235$ & 0.422 & $22.4017 \pm 0.0552$ & $-1.1912 \pm 0.4730$ & $-0.0813 \pm 0.0652$ & $14.07 \pm 2.46$ & 2 \\
\hline $\mathrm{f} 244$ & 0.540 & $23.2981 \pm 0.0524$ & $-0.5891 \pm 0.7566$ & $-0.0055 \pm 0.0801$ & $16.24 \pm 3.27$ & 2 \\
\hline $1997 \mathrm{G}$ & 0.763 & $24.2807 \pm 0.2579$ & $-0.8583 \pm 1.3793$ & $-0.4161 \pm 0.2310$ & $7.08 \pm 3.37$ & 3 \\
\hline $1997 \mathrm{~S}$ & 0.612 & $23.4756 \pm 0.0639$ & $3.6292 \pm 1.7033$ & $0.1103 \pm 0.1169$ & $21.70 \pm 2.72$ & 3 \\
\hline 1997af & 0.579 & $23.5343 \pm 0.0796$ & $-1.0871 \pm 0.4901$ & $-0.2081 \pm 0.1852$ & $7.78 \pm 3.04$ & 3 \\
\hline 1997ai & 0.454 & $22.8788 \pm 0.0627$ & $-2.6928 \pm 0.9158$ & $-0.0040 \pm 0.0787$ & $21.42 \pm 2.74$ & 3 \\
\hline 1997aj & 0.581 & $23.1959 \pm 0.0822$ & $0.7138 \pm 0.7974$ & $0.1991 \pm 0.1123$ & $6.23 \pm 2.60$ & 3 \\
\hline 1997ap & 0.831 & $24.3496 \pm 0.0633$ & $0.3579 \pm 0.5601$ & $-0.0115 \pm 0.0384$ & $17.21 \pm 3.82$ & 3 \\
\hline $2000 \mathrm{fr}$ & 0.543 & $23.0376 \pm 0.0263$ & $0.7208 \pm 0.2533$ & $-0.0359 \pm 0.0281$ & $7.81 \pm 2.70$ & 4 \\
\hline $2001 \mathrm{gm}$ & 0.478 & $\cdots$ & $\cdots$ & $\cdots$ & $15.52 \pm 7.74$ & 4 \\
\hline 2001go & 0.552 & $\ldots$ & $\ldots$ & $\ldots$ & $27.15 \pm 4.34$ & 4 \\
\hline $2001 \mathrm{gw}$ & 0.363 & $\cdots$ & $\cdots$ & $\cdots$ & $9.57 \pm 3.77$ & 4 \\
\hline 2001gy & 0.511 & $\cdots$ & $\cdots$ & $\cdots$ & $3.66 \pm 3.23$ & 4 \\
\hline 2001ha & 0.58 & $\cdots$ & $\cdots$ & $\cdots$ & $21.80 \pm 5.40$ & 4 \\
\hline $2001 \mathrm{hc}$ & 0.35 & $\cdots$ & $\cdots$ & $\cdots$ & $7.28 \pm 2.93$ & 4 \\
\hline $2002 \mathrm{gl}$ & 0.510 & $\cdots$ & $\cdots$ & $\cdots$ & $16.25 \pm 3.30$ & 4 \\
\hline
\end{tabular}


Table 4. continued.

\begin{tabular}{llrrrrc}
\hline \hline SN name & $z_{\mathrm{CMB}}$ & $m_{B}$ & $x_{1}$ & $c$ & $E W_{w}\{$ Si II $\}$ & Reference \\
\hline $2002 \mathrm{~km}$ & 0.606 & $\cdots$ & $\cdots$ & $\cdots$ & $15.05 \pm 4.37$ & 4 \\
$2002 \mathrm{ks}$ & 1.181 & $\ldots$ & $\cdots$ & $\cdots$ & $20.79 \pm 6.45$ & 4 \\
$1999 \mathrm{ff}$ & 0.455 & $23.2026 \pm 0.0516$ & $-1.4400 \pm 0.6801$ & $-0.0109 \pm 0.0698$ & $21.45 \pm 8.25$ & 5 \\
$1999 \mathrm{fh}$ & 0.369 & $\ldots$ & $\cdots$ & $\cdots$ & $18.47 \pm 2.76$ & 5 \\
$1999 \mathrm{fj}$ & 0.816 & $24.1845 \pm 0.0621$ & $0.2386 \pm 0.5735$ & $-0.0833 \pm 0.1807$ & $16.82 \pm 3.33$ & 5 \\
$1999 \mathrm{fk}$ & 1.057 & $24.7553 \pm 0.0609$ & $-0.2806 \pm 0.9992$ & $-0.0586 \pm 0.0707$ & $14.60 \pm 6.08$ & 5 \\
$1999 \mathrm{fm}$ & 0.950 & $24.2363 \pm 0.0445$ & $1.5816 \pm 0.4610$ & $0.0279 \pm 0.0904$ & $5.81 \pm 7.05$ & 5 \\
$1999 \mathrm{fn}$ & 0.477 & $22.7513 \pm 0.0393$ & $0.7821 \pm 0.3983$ & $0.0230 \pm 0.0415$ & $12.51 \pm 2.98$ & 5 \\
$1999 \mathrm{fw}$ & 0.278 & $21.6893 \pm 0.0671$ & $-1.2738 \pm 0.6348$ & $0.0975 \pm 0.0616$ & $12.85 \pm 2.84$ & 5 \\
\hline
\end{tabular}

References: (1) Howell et al. (2005); (2) Matheson et al. (2005); (3) Hook et al. (2005); (4) Lidman et al. (2005); (5) Tonry et al. (2003).
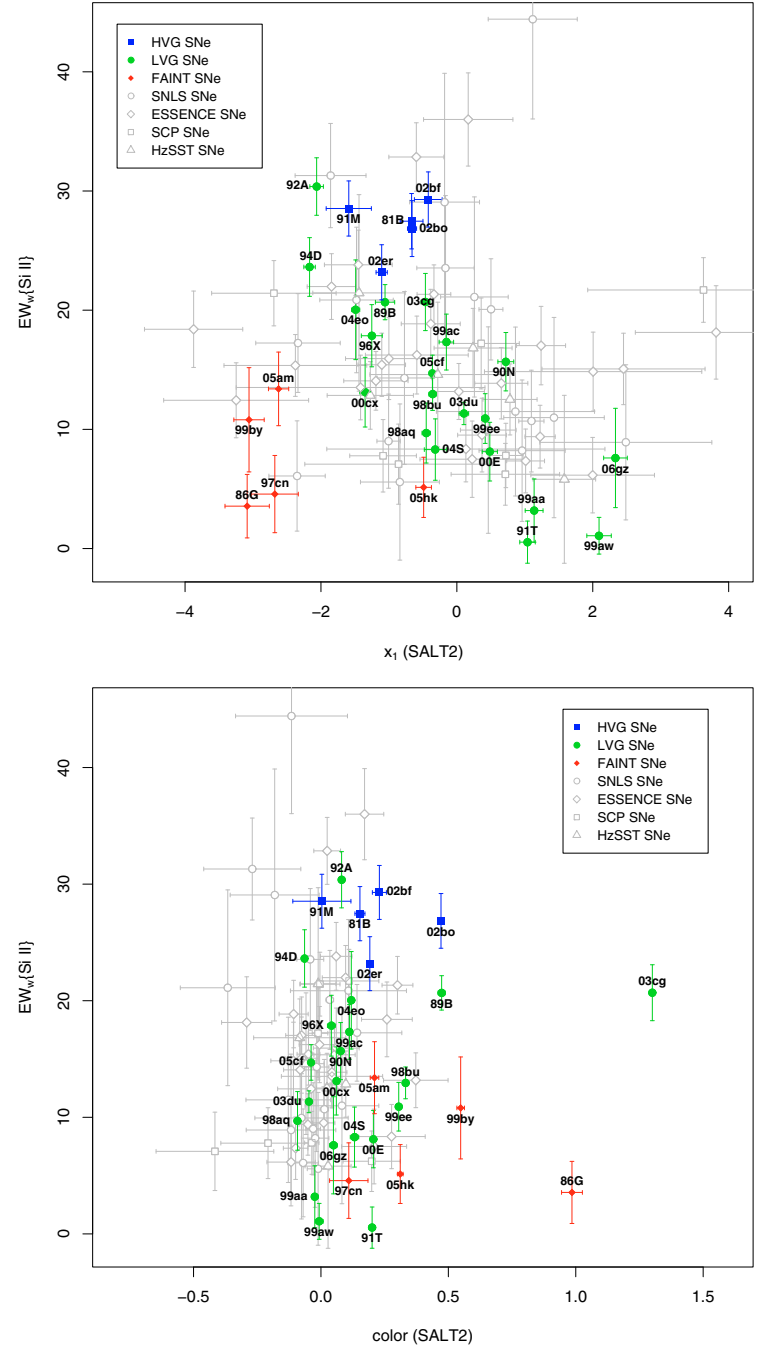

Fig. 4. $E W_{w}\{$ Si II $\}$ versus $x_{1}$ (top) and colour (bottom) estimated using SALT2 including high- $z$ SNe. The colours chosen for different sub-classes are the same as in Benetti et al. (2005); Pastorello et al. (2007b): blue filled squares for HVG SNe, light green filled circles for LVG SNe and red symbols for FAINT SNe.

difference in kinetic energy of the ejecta (of the order of $\sim 2 \%)^{7}$. This difference in kinetic energy implies that HVG SNe lightcurves are narrower than LVG SNe ones. Even if we assume that it is the only difference between these two groups of SNe, this

\footnotetext{
7 Although this difference is too small to explain the spectral diversity between the HVG and LVG SNe.
}

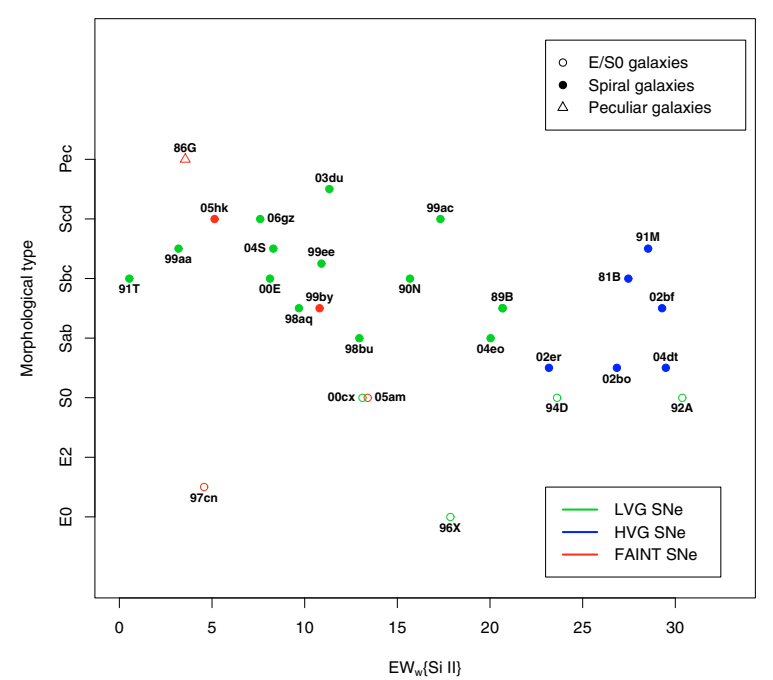

Fig. 5. Morphological type of the $\mathrm{SN}$ host galaxy versus measured $E W_{w}\{\mathrm{Si}$ II $\}$ for nearby SNe.

might cause an intrinsic dispersion in the luminosity/light-curve shape relation.

As shown in Fig. 4, we also find 4 events considered as non-standard in Pastorello et al. (2007b); Branch et al. (2006); Hachinger et al. (2006), namely SNe 1989B, 1991M, 1992A and 2004eo. Among them, SN 1991M is labeled as HVG SN, while the others are shown as LVG objects. We may add to this list an HVG SN 2002er that has been found to have properties common to both HVG and LVG SNe, like a lower expansion velocity but temperatures higher than of other HVG SNe (Benetti et al. 2005; Tanaka et al. 2008). These few events can be considered as transitional objects, linking all three sub-classes and providing continuity between the groups. We may also include SN 1994D in these objects as the top panel in Fig. 4 indicates; in addition, SN 2005hk seems to establish a link between the LVG and FAINT SNe. Similar plots like in Fig. 4 of $E W_{w}\{\mathrm{Si} \mathrm{II}\}$ versus $\Delta m_{15}$ or stretch confirm a strong affinity of this supernova for the FAINT group.

Another object of interest is the peculiar SN 2006gz (an SN with the slowest fading light-curves ever seen in a SN Ia), whose properties deviate from the LVG SNe. Its early-time Si II velocity is low, like for LVG SNe, which is attributed to an envelope of unburned carbon that slows expansion (see Hicken et al. 2007).

A linear fit in upper plot in Fig. 4, with only LVG SNe at low $-z$ included, gives:

$E W_{w}\{\mathrm{Si} \mathrm{II}\}=(12.21 \pm 0.32)-(5.88 \pm 0.30) x_{1}$ 
We looked for the SN host galaxy type to explore its effect on the SN sub-class. It was emphasized by Hamuy et al. (1996); Altavilla et al. (2004); Gallagher et al. (2005) that intrinsically faint events rather occur in E/S0 galaxies. The diagram in Fig. 5 points to the conclusion that early-type (E/S0) galaxies lack $\mathrm{SNe}$ with smaller $E W_{w}\{\mathrm{Si} \mathrm{II}\}$ values, say $\leq 12$. Among our sample, no HVG SN hosted in the elliptical galaxy is found, though there are two ambiguous SNe between LVG and HVG group in S0 galaxies. These two are identified as SNe 1992A and 1994D.

Regarding the issue of whether or not the distance estimator varies across different environments, Conley et al. (2006) suggest that stretch correction works well no matter the SN environment. On the other hand, it is not the case with colour, since this correction accounts for dust or extinction effects and also for the intrinsic SN relationship (see, e.g. Sullivan et al. 2006; Conley et al. 2007).

\subsection{High-z SNe}

The method presented in this work allows us to examine in a consistent way low and high- $z \mathrm{SNe}$ for which we calculated $E W_{w}\{\mathrm{Si}$ II $\}$ and SALT2 light-curve parameters.

Adding high- $z$ SNe to the cluster analysis one obtains the following SNe that are classified as HVG SNe: 03D4fd, 04D3dd, 04D3gx, b013 and d058.

Two outliers with the largest $x_{1}$ values can also be noticed in upper panel of Fig. 4, namely SCP SN 1997S and ESSENCE d033. In the opposite region of lower $x_{1}$ values, besides SN e138, there are a few candidates, SCP 1997ai SNLS-03D4cz, 03D4cn and ESSENCE f216, e029 that seem to have affinities towards the group of FAINT SNe.

ESSENCE SN d117 is identified in the ambiguous zone between FAINT and LVG SNe. Further, it is hard to classify SNe 03D1fq, 03D4fd, e132 and f011.

We do not exclude the possibility that dispersion seen in upper plot in Fig. 4 is due to host galaxy contamination, especially for the SNLS and ESSENCE SNe. As it is mentioned in 2.3 the presence of galaxy light in the SN spectra might lead to underestimated values of $E W_{w}\{\mathrm{Si} I I\}$.

The bottom panel in Fig. 4 indicates that the correlation between $E W_{w}\{\mathrm{Si}$ II $\}$ and $c$ is quite weak, but there are outliers. It can justify an eventual colour cut, say $-0.2<c<0.2$, that would ensure a more homogeneous SN data set for cosmological use. Indeed, $\mathrm{SNe}$ with $c$ out of the mentioned interval tend to have more dispersion in the Hubble diagram (see Arsenijevic 2008; Kowalski et al. 2008).

In addition, we find no significant correlation between $E W_{w}\{\mathrm{Si}$ II $\}$ and the absolute magnitude $M_{B}$ corrected for $x_{1}$ and $c$, using updated values for $\alpha$ and $\beta$ from Guy et al. (2007), although there are many outliers present. The linear factor in the relation $M_{B_{\text {corr }}} \sim E W_{w}\{\mathrm{Si} \mathrm{II}\}$ for the low- $z \mathrm{SNe}$ is of the order of 0.01 . We used values of the distance modulus $\mu$ for low $-z$ SNe from Tammann et al. (2008). These questions are further discussed in Arsenijevic (2008).

A source of systematic errors in the Hubble diagram comes from uncertainties in distance or host galaxy reddening or from intrinsic properties of the SNe. With the available data we are not yet able to distinguish between potential systematic effects that come from magnitude dependence on intrinsic colour and the colour contribution due to dust effects in the host galaxy of the SNe. Note that in order to examine the dust properties of distant galaxies using $\mathrm{SNe} \mathrm{Ia}$, one needs to first remove the intrinsic dependence of SNe luminosity on the light-curve parameters.
An important issue in cosmology with $\mathrm{SNe}$ Ia is how to clean the SN sample of any objects that might not obey a standard peak luminosity/light-curve shape relation. Our results confirm the possible contamination of the high- $z$ sample with HVG SNe. This fact deserves further attention as a potential source of systematic error, as was also suggested in Tanaka et al. (2008).

Previous comparisons between low and high- $z$ SNe spectral features have shown the homogeneity within the two samples. We have taken a step further by introducing a new approach that allows us to identify SN Ia sub-classes, independent of the SN redshift, using only one spectrum with Si II 4000 together with the light-curve width and colour parameters.

\section{Conclusions}

The use of SNe Ia for cosmology relies on empirical calibration techniques on the light-curves and K-correction. It would be reassuring to have a usable indicator measured directly from $\mathrm{SN}$ spectra that can be used as an independent calibrator of the luminosity of an SN Ia event. Applying a straightforward transformation using wavelets, we were able to estimate, in a consistent manner on a fair number of SNe Ia, the equivalent width of the Si II 4000 feature which previously showed potential use for cosmology. At low redshift, we were able to automatically distinguish three classes of SNe Ia previously found by other authors using much more information, such as the expansion velocity gradient.

The same wavelet-based approach was applied to high redshift data, revealing an analogous clustering tendency to that found for low redshift supernovae. Yet it is not clear whether the three sub-classes, found in the low $z$ and verified in the high- $z$ sample, are completely distinct or come from one continuous family. If so, this could possibly be the remnant of an extra parameter or a more complex modelling of the supernova data. The implementation of our method using recently available much larger spectral samples and the use of different SN Ia sub-classes in cosmological analysis is now in preparation.

Although the method presented here is promising and we are planning to apply it to other spectral features, we remark that line ratios, pseudo-equivalent widths and their current derivatives are not optimal to extract information on lower signal-to-noise spectra. Parameters calculated from spectral features, such as equivalent widths, still consider only local information and each feature independently. New indicators based on combining local information, such as wavelet coefficients from the whole spectra, can provide a better characterisation of the supernovae.

Acknowledgements. This work was supported by Fundação para a Ciência e Tecnologia (FCT), Portugal under POCTI/CTE-AST/57664/2004. V. Arsenijevic acknowledges support from FCT under grant no. SFRH/BD/11119/2002 and S. Fabbro grant no. SFRH/BPD/31817/2006. Most of the code was written in $\mathrm{R}^{8}$. We would like to thank $\mathrm{G}$. Nason for useful comments on the implementation of wavethresh package.

\section{References}

Altavilla, G., Fiorentino, G., Marconi, M., et al. 2004, MNRAS, 349, 1344 Altavilla, G., Stehle, M., Ruiz-Lapuente, P., et al. 2007, A\&A, 475, 585A Anupama, G. C., Sahu, D. K., \& Jose, J. 2005, A\&A, 429, 667 Arsenijevic, V. 2008, Ph.D. Thesis, Technical University of Lisbon, in preparation

8 R Development Core Team (2007). R: A language and environment for statistical computing. $\mathrm{R}$ Foundation for Statistical Computing, Vienna, Austria. ISBN 3-900051-07-0, URL http:// wWw.r-project.org 
Astier, P., Guy, J., Regnault, N., et al. 2006, A\&A, 447, 31

Barbon, R., Benetti, S., Rosino, L., Cappellaro, E., \& Turatto, M. 1990, A\&A, 237, 79

Benetti, S., et al. 2005, ApJ, 623, 1010

Benetti, S., Meikle, P., Stehle, M., et al. 2004, MNRAS, 348, 261

Benetti, S., Cappellaro, E., Turatto, M., et al. 2006, ApJ, 653, L129

Blondin, S., Dessart, L., Leibundgut, B., et al. 2006, AJ, 131, 1648

Bongard, S., Baron, E., Smadja, G., Branch, D., \& Hauschildt, P. H. 2006, ApJ, 647,513

Branch, D., Lacy, C. H., McCall, M. L., et al. 1983, ApJ, 270, 123

Branch, D., Garnavich, P., Matheson, T., et al. 2003, AJ, 126, 1489

Branch, D., Baron, E., Thomas, R. C., et al. 2004, PASP, 116, 903

Branch, D., Dang, L. C., Hall, N., et al. 2006, PASP, 118, 560

Bronder, J., Hook, I. M., Astier, P., et al. 2008, A\&A, 477, 717

Brown, P. J., Holland, S. T., James, C., et al. 2005, ApJ, 635, 1192

Cardelli, J. A., Clayton, G. C., \& Mathis, J. S. 1989, ApJ, 345, 245

Conley, A., Howell, D. A., Howes, A., et al. 2006, ApJ, 132, 1707

Conley, A., Carlberg, R. G., Guy, J., et al. 2007, ApJ, 664, L13

Elias-Rosa, N., Benetti, S., Cappellaro, E., et al. 2006, MNRAS, 369, 1880

Ellis, R. S., Sullivan, M., Nugent, P. E., et al. 2008, ApJ, 674, 51

Filippenko, A. V. 1997, ARA\&A, 35, 309

Folatelli, G. 2004, New Astron. Rev., 48, 623

Gallagher, J. S., Garnavich, P. M., Berlind, P., et al. 2005, ApJ, 634, 210

Garavini, G., Folatelli, G., Goobar, A., et al. 2004, AJ, 128, 387

Garavini, G., Aldering, G., Amadon, A., et al. 2005, AJ, 130, 2278

Garavini, G., Folatelli, G., Nobili, S., et al. 2007a, A\&A, 470, 411

Garavini, G., Nobili, S., Taubenberger, S., et al. 2007b, A\&A, 471, 527

Garnavich, P. M., Bonanos, A. Z., Krisciunas, K., et al. 2004, ApJ, 613, 1120

Gomez, G., \& Lopez, R. 1998, AJ, 115, 1096

Guy, J., Astier, P., Baumont, S., et al. 2007, A\&A, 466, 11

Guy, J., Astier, P., Nobili, S., Regnault, N., \& Pain, R. 2005, A\&A, 443, 781

Hachinger, S., Mazzali, P. A., \& Benetti, S. 2006, MNRAS, 370, 299

Hamuy, M., Maza, J., Pinto, P. A., et al. 2002, AJ, 124, 417

Hamuy, M., Phillips, M. M., Suntzeff, N. B., et al. 1996, AJ, 112, 2391

Hicken, M., Garnavich, P. M., Prieto, J. L., et al. 2007, ApJ, 669, L17

Hook, I. M., Howell, D. A., Aldering, G., et al. 2005, AJ, 130, 2788

Howell, D. A., Sullivan, M., Perrett, K., et al. 2005, ApJ, 634, 1190

Howell, D. A., Sullivan, M., Nugent, P., et al. 2006, Nature, 443, 309

James, J. B., Davis, T. M., Schmidt, B. P., \& Kim, A. G. 2006, MNRAS, 370, 933

Jeffery, D. J., Leibundgut, B., Kirshner, R. P., et al. 1992, ApJ, 397, 304

Jha, S., Garnavich, P. M., Kirshner, R. P., et al. 1999, ApJS, 125, 73

Jha, S., Riess, A. G., \& Kirshner, R. P. 2007, ApJ, 659, 122

Kirshner, R. P., Jeffery, D. J., Leibundgut, B. et al. 1993, ApJ, 415, 589

Kotak, R., Meikle, W. P. S., Pignata, G., et al. 2005, A\&A, 436, 1021

Kowalski, M., et al. 2008, ApJ, accepted [arXiv : 0804 . 4142]

Krisciunas, K., Garnavich, P. M., Stanishev, V., et al. 2007, AJ, 133, 58
Leonard, D. C., Li, W., Filippenko, A. V., \& Foley, R. J., Chornock, R. 2005, ApJ, 632, 450

Li, W., Qiu, Y. L., Qiao, Q. Y., et al. 1999, AJ, 117, 2709

Li, W., Filippenko, A. V., Gates, E., et al. 2001, PASP, 113, 1178

Li, W., Filippenko, A. V., Chornock, R., et al. 2003, PASP, 115, 453

Lidman, C., Howell, D. A., Folatelli, G., et al. 2005, A\&A, 430, 843

Mallat, S. G. 1989, IEEE Trans. PAMI, 11, 674

Matheson, T., Blondin, S., Foley, R. J., et al. 2005, AJ, 129, 2352

Mazzali, P. A., Lucy, L. B., Danziger, I. J., et al. 1993, A\&A, 269, 423

Mazzali, P. A., Danziger, I. J., \& Turatto, M. 1995, A\&A, 297, 509

Miknaitis, G., Pignata, G., Rest, A., et al. 2007, ApJ, 666, 674

Nason, G. P., \& Silverman, B. W. 1994, J. Comp. Graph. Statist., 3, 163

Nugent, P., Phillips, M., Baron, E., Branch, D., \& Hauschildt, P. 1995, ApJ, 455, L147

Pastorello, A., Taubenberger, S., Elias-Rosa, N., et al. 2007a, MNRAS, 376, 1301

Pastorello, A., Mazzali, P. A., Pignata, G., et al. 2007b, MNRAS, 377, 1531

Patat, F., Benetti, S., Cappellaro, E., et al. 1996, MNRAS, 278, 111

Pei, Y. C. 1992, ApJ, 395, 130

Perlmutter, S., Aldering, G., Goldhaber, G., et al. 1999, ApJ, 517, 565

Phillips, M. M., Phillips, A. C., Heathcote, S. R., et al. 1987, PASP, 99, 592

Phillips, M. M., Wells, L. A., Suntzeff, N. B., et al. 1992, AJ, 103, 1632

Phillips, M. M., Krisciunas, K., Suntzeff, N. B., et al. 2006, AJ, 131, 2615

Phillips, M. M., Li, W., Frieman, J. A., et al. 2007, PASP, 119, 360

Pignata, G., Patat, F., Benetti, S., et al. 2004, MNRAS, 355, 178

Prieto, J. L., et al. 2007, [arXiv:0706.4088]

Prieto, J. L., Stanek, K. Z., \& Beacom, J. F. 2008, ApJ, 673, 999

Quimby, R., Höflich, P., \& Wheeler, C. J. 2007, ApJ, 666, 1083

Riess, A. G., Filippenko, A. V., Challis, P., et al. 1998, AJ, 116, 1009

Sahu, S., Tanaka, M., Anupama, G. C., et al. 2008, ApJ, 680, 580

Salvo, M. E., Cappellaro, E., Mazzali, P. A., et al. 2001, MNRAS, 321, 254

Stanishev, V., Goobar, A., Benetti, S., et al. 2007, A\&A, 469, 645

Starck, J.-L., Siebenmorgen, R., \& Gredel, R. 1997, ApJ, 482, 1011

Strolger, L. G. 2002, AJ, 124, 2905

Sullivan, M., Le Borgne, D., Pritchet, C. J., et al. 2006, ApJ, 648, 868

Tammann, G. A., Sandage, A., \& Reindl, B. 2008

[arXiv: 0806.3018]

Tanaka, M., Mazzali, P. A., Benetti, S., et al. 2008, ApJ, 677, 448

Tonry, J. L., Schmidt, B. P., Barris, B., et al. 2003, ApJ, 594, 1

Trundle, C., Kotak, R., Vink, J. S., \& Meikle, W. P. S. 2008, A\&A, 483, 47

Turatto, M., Benetti, S., Cappellaro, E., et al. 1996, MNRAS, 283, 1

Turatto, M., Piemonte, A., Benetti, S., et al. 1998, AJ, 116, 2431

Valentini, G., Di Carlo, E., Massi, F., et al. 2003, AJ, 595, 779

Wang, L., Baade, D., Höflich, P., et al. 2004, ApJ, 604, L53

Wang, L., Baade, D., \& Patat, F. 2007, Science, 315, 212

Wang, L., \& Wheeler, C. J. 2008, ARA\&A, 46, 433

Weingartner, J. C., \& Draine, B. T. 2001, ApJ, 548, 296

Wells, L. A., Phillips, M. M., Suntzeff, B., et al. 1994, AJ, 108, 2233 